\title{
Drawing up an individual risk index for development of metachronous neoplastic lesions in resected colorectal cancer
}

\author{
Ana Borda ${ }^{1}$, José María Martínez-Peñuela ${ }^{2}$, Fernando Borda ${ }^{1}$, Miguel Muñoz-Navas ${ }^{3}$, \\ Francisco Javier Jiménez and Cristina Carretero ${ }^{3}$ \\ ${ }^{1}$ Departments of Gastroenterology and ${ }^{2}$ Pathology. Complejo Hospitalario de Navarra (A). \\ ${ }^{3}$ Department of Gastroenterology. Clínica Universidad de Navarra. Pamplona, Spain
}

\begin{abstract}
Aim: to identify possible risk factors for the development of metachronous lesions in colorectal cancer $(\mathrm{CRC})$ which would allow to establish a post-surgical individual prognostic index.

Patients and methods: three hundred eighty-two surgically treated CRC were reviewed. We compared the incidence of metachronous lesions in 40 variables concerning patient clinical data and initial neoplastic findings. An individual risk index for metachronicity was drawn up including those variables which presented significant differences in multivariate logistic regression, dividing patients into three groups.

Results: variables with prognostic value for metachronicity were distal cancer location: $\mathrm{OR}=2.30$ (1.03-5.13), alcohol intake: OR $=2.20$ (1.08-4.48), presence of synchronous adenomas: isolated: $\mathrm{OR}=2.47$ (1.03-4.48), multiple: $\mathrm{OR}=4.26$ (1.78-10.17), and presence of synchronous advanced adenoma: $\mathrm{OR}=2.91$ (1.5212.60). Tumor MUC-5 expression proved to have a protective role: $\mathrm{OR}=0.23(0.08-0.66)$. An individual risk score was established considering these variables and patients could be classified into three groups, with a discrimination power for metachronicity of $p<$ 0.0000001 . Classification in high and low risk groups had a sensitivity $=75.32 \%$, specificity $=84.21 \%$, positive predictive value $=$ $75.34 \%$, negative predictive value $=92.31 \%$ and global diagnostic accuracy $=80.75 \%$.

Conclusions: the identification of risk factors for the development of metachronous lesions allow to calculate, at the time of surgical treatment, an individual prognostic index and to classify patients into three different risk groups. In high and low risk groups, both specificity and accuracy were acceptable for the prognosis of metachronous lesions, being remarkable the negative predictive power of our classification, which could become relevant when planning a different endoscopic follow up of these patients.
\end{abstract}

Key words: Colorectal cancer. Follow-up. Metachronous lesions.

Received: $10-11-2012$

Accepted: 13-03-2012

Correspondence: Ana Borda. Department of Gastroenterology. Complejo Hospitalario de Navarra (A). c/ Irunlarrea, s/n. 31008 Pamplona, Navarra. Spain e-mail: abordamartin@yahoo.es
Borda A, Martínez-Peñuela JM, Borda F, Muñoz-Navas M, Jiménez FJ, Carretero C. Drawing up an individual risk index for development of metachronous neoplastic lesions in resected colorectal cancer. Rev Esp Enferm Dig 2012; 104: 291-297.

\section{INTRODUCTION}

The majority of colorectal cancers (CRC) are sporadic cases $(1,2)$ and little is known about metachronous or synchronous neoplasms development $(3,4)$. These multicentric lesions could be due to mutations in genes related to apoptosis, cellular proliferation or DNA repairing (5), or forms of hereditary non polyposis colorectal cancer syndrome with low penetrance (6). Finally, this tumor multicentricity could be caused by multiple interacting factors, such as personal or familial predisposition, environmental mechanisms, morphological or immunophenotypical tumor features which would favoured an increased risk of neoplasm development along different colonic sites (7). Once the tumor and all the possible synchronic lesions are endoscopically or surgically resected, the tumor multicentricity might promote the development of new neoplastic lesions $(8,9)$. Similar to synchronous lesions, metachronic neoplasms will be adenomas in most cases and less frequently CRC. There are several publications related to development of metachronous lesions after the resection of the initial polyps in patients with adenomas, but without CRC (10-14). However, results obtained from these studies are frequently discordant variables depending on patients and adenomas features. Publications regarding possible risk factors for metachronous lesions development in resected CRC are scarce (8,13-16). Furthermore, their analysis included a limited number of variables and drawn discordant conclusions. On the other hand, some authors suggest CRC history as a possible risk factor favoring metachronous lesion development $(17,18)$. Consequently, CRC patients could represent, from the beginning, a high risk population for having 
metachronous lesions, which could bias the results registered in series of patients with adenomas, but with no CRC.

The study of possible differences between CRC cases, with and without metachronous lesions, could determine some risk factors for new lesions development.

Nowadays, there is no agreement about which evolutive control might be better, after CRC is resected and different Clinical Guidelines have been proposed for this purpose (19-21). Early identification of these risk parameters for developing metachronic lesions could be useful to classify patients in subgroups of high or low probability of having new neoplasms, which could allow a more or less closer follow-up after all the polyps are endoscopically removed and CRC is resected (22).

\section{PATIENTS AND METHODS}

Three hundred eighty-two CRC diagnosed in two hospitals of our city by pre-, intra- or postoperative total colonoscopy from January 1999 to December 2003, were reviewed. All the synchronous lesions and the primary carcinoma were resected with curative intention and examined by the same pathologist. Multiple polyposis syndromes and intestinal inflammatory disease cases were excluded. Presence of another colorectal tumor at the moment of diagnosis and tubular, villous, or tubulo-villous adenomas were considered as synchronous neoplastic lesions, without including hyperplastic polyps. In CRC cases, metachronous lesion was defined as the lesion that appeared at least 12 months after resection and was not located in the surgical anastomosis (23). Follow-up was performed by total colonoscopy excluding all cases with an outcome inferior to 12 months.

Patients or their relatives in cases of death answered a postal survey authorizing to be included in the study and where all clinical data were filled out.

Our series was classified into two groups according to the presence of metachronous lesions during follow-up. Differences between two groups related to the following parameters were studied:

- Patients characteristics: age ( $<60$ or $\geq 60$ years); body mass index (BMI) $(<21 ; 21-24.9,25-29.9, \geq$ 30); alcohol and tobacco consumption (yes/no); acetylsalicylic intake for at least one year; personal history of: CRC, cancer of any other location, colorectal adenoma; fist-degree relative with any cancer, colorectal adenomas, Amsterdam I and II criteria $(24,25)$ or Revised 2004 Bethesda Guidelines (26).

- Initial neoplastic lesion features: presence or not of synchronous CRC; initial cancer location; initial obstructive cancer; histological cancer type; differentiation grade; pTNM stage (27); presence and synchronous adenomas location; presence or not of advanced synchronous adenoma.

- Immunohistochemical CRC features: immunostaining was evaluated by a tissue micro arrays method, using antibodies against several markers related to $\mathrm{CRC}$ aggressiveness. These markers were: DNA repair genes: MLH-1, MSH-2, MLH-6; tumor suppressor genes: p53; apoptosis regulator genes: Bcl-2; epithelial cytoskeletal markers: cytokeratin-7 and cytokeratin20; mucins: MUC-1, MUC-2, MUC-5, MUC-6; tumor antigens: CEA; COX-2.

Univariable analysis was performed using $t$-student and Mann Whitney tests for quantitative variables and Chisquared and Fisher for qualitatives. Subsequently, a multivariable analysis with non conditional multiple regression method was carried out.

Presence or not of metachronous lesions was considered as the dependent variable, whereas variables that achieved statistical significance $(\mathrm{p}<0.05)$ in the univariate analysis were considered independent.

\section{Drawing up an individual risk index}

The model obtained from the multivariable analysis was used to draw up a prognostic risk index for developing metachronous lesions, divided into 3 categories: low, medium and high risk. The index was calculated with the beta coefficient corresponding to each variable in the multivariable regression analysis, multiplied by 1.5 and rounded up to obtain a result with no decimals.

Each patient score was calculated according to the number of variables presented, included in our prognostic index. Once this score is obtained, the most adequate cut-off points to stratify our patients in 3 risk groups for developing metachronous lesions were determined. For this purpose, all the possible groups were considered and the discrimination power of each category was calculated by Chi Square test, selecting the most statistically significant subgroup. The frequency of metachronous lesions in the medium risk subgroup was compared to the overall series.

Afterwards, considering the high risk group as a positive test and the low risk group as a negative test sensibility, specificity, positive predictive value and negative predictive value, and prognostic accuracy with a $95 \%$ confidence interval was determined.

The medium risk group was considered as a "non conclusive" result, thus it was no included in the analysis.

\section{RESULTS}

Three hundred eighty-two patients with resected CRC were studied. The following synchronous neoplastic lesions were registered at the moment of diagnosis: 28 carcinomas, which mean $7.3 \%$ (26 CRC were double and 2 triple), all associated with adenomas.

The endoscopic median follow-up was 48 months and 2.74 \pm 1.47 colonoscopies per patient were performed. In the overall results, 7 metachronous carcinomas were diagnosed (1.8\%) and metachronous adenomas in 162 cases (42.4\%). Twenty 
Table I. Significant variables in multivariate logistic regression analysis for metachronous lesions developing

\begin{tabular}{|c|c|c|c|}
\hline Variable & Beta & $p$ & Odds ratio IC 95\% \\
\hline Distal cancer location & 0.83 & 0.042 & $2.30(1.03-5.13)$ \\
\hline Alcohol intake & 0.79 & 0.03 & $2.20(1.08-4.48)$ \\
\hline \multicolumn{4}{|l|}{ Synchronous adenoma } \\
\hline Isolated & 0.90 & 0.041 & $2.47(1.03-4.48)$ \\
\hline Multiple & 1.45 & 0.001 & $4.26(1.78-10.17)$ \\
\hline $\begin{array}{l}\text { Synchronous advanced } \\
\text { adenoma }\end{array}$ & 1.07 & 0.037 & $2.91(1.52-12.60)$ \\
\hline MUC-5 expression & 1.48 & 0.006 & $0.23(0.08-0.66)$ \\
\hline
\end{tabular}

\section{\% METACHRONOUS LESIONS}

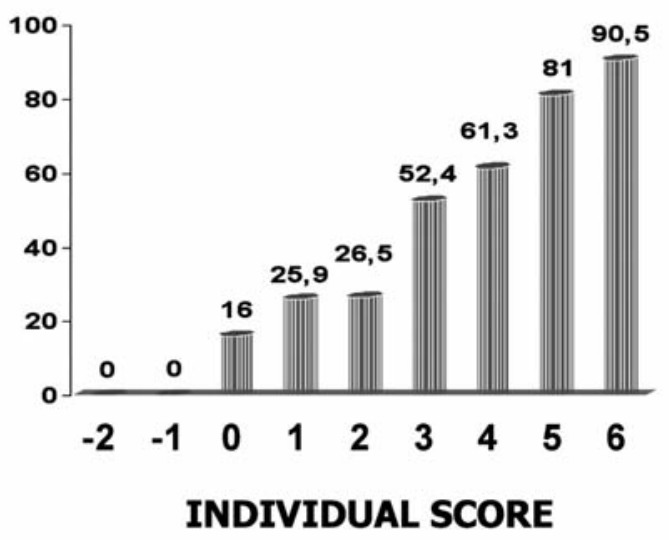

Fig. 1. Individual score and frequency of metachronous lesions.

four patients (6.3\%) developed advanced metachronous adenomas. All metachronous cancers were associated with metachronous adenomas. The average time to diagnosis of the first metachronous lesion was $28.4 \pm 21.8$ months. Diagnosis was performed after a 5 years follow-up in 15 of 162 cases with metachronous lesions $(9.3 \%)$.

After the multivariate analysis was carried out, the number of cases was reduced to 251 patients. Five of the 40 studied parameters showed statistical significant differences related to the frequency for developing metachronous lesions. Hence, these variables could be considered as independent risk factors for developing metachronicity: distal cancer location, alcohol intake, presence of isolated or multiple synchronous adenoma, synchronous advanced adenoma and tumor MUC-5 expression (Table I).

The score for every variable in the individual prognostic index is detailed in table II and figure 1. Starting from this score, patients were included in the following risk groups for developing metachronous neoplasms: low risk: from 2 to +1 points ( $45.5 \%$ of cases); medium risk: from 2 to 3 points ( $25.5 \%$ of cases); high risk: from 4 to 6 points $(29.1 \%$
Table II. Prognostic index score

\begin{tabular}{ll}
\hline Variable & Score \\
\hline Initial cancer location & \\
$\quad$ Proximal & 0 \\
Distal & 1 \\
Alcohol intake & \\
$\quad$ Non drinkers & 0 \\
$\quad$ Drinkers & 1 \\
Synchronous adenomas & \\
None & 0 \\
Isolated & 1 \\
Multiple & 2 \\
Synchronous advanced adenoma & \\
No & 0 \\
Yes & 2 \\
MUC-5 expression & \\
No & 0 \\
Yes & -2 \\
\hline
\end{tabular}

Table III. Risk groups and frequency of metachronous lesions (multivariate analysis, $\mathrm{n}=\mathbf{2 5 1}$ )

\begin{tabular}{lcc}
\hline Risk groups & $\begin{array}{c}\text { No metachronous } \\
\text { lesion } \\
n(\%)\end{array}$ & $\begin{array}{c}\text { Metachronous } \\
\text { lesion } \\
n(\%)\end{array}$ \\
\hline Low risk & $96 / 114(84.2)$ & $18 / 114(15.8)$ \\
Medium risk & $36 / 64(56.3)$ & $28 / 64(43.7)$ \\
High risk & $18 / 73(24.7)$ & $55 / 73(75.3)$ \\
\hline
\end{tabular}

$p<0.0000001$.

of cases). Differences in metachronous lesions frequency between the 3 risk groups were statistically significant with a $p<0.0000001$ and are shown in table III.

The frequency of metachronous adenomas in the medium risk group (43.7\%) was compared to the metachronous lesions rate in the global series $(42.2 \%)$, with a nearly identical result: $p=0.90$. On account of this, the medium risk group could be considered similar to the global group, and thereby, as a "non conclusive" result.

If we analyze the frequency of metachronous lesions between the low risk group (15.8\%) and the high risk group $(75.3 \%)$, a high statistical significance is obtained [p < 0.0000001; OR = 16.30; $($ CI 95\% = 7.39-36.51) $]$.

Sensibility, specificity, positive predictive value and negative predictive value and diagnostic accuracy for low and high risk groups was determined, excluding the medium risk group from the analysis. High risk group was considered as a "positive test", and low risk group as a "negative test" for developing metachronous lesions. Results are shown in table IV. 
Table IV. Sensibility, specificity, positive and negative predictive value and diagnostic accuracy of the prognostic index score

\begin{tabular}{lll}
\hline & Value (\%) & IC (95\%) \\
\hline Sensibility & 75.32 & $(64.77-85.91)$ \\
Specificity & 84.21 & $(77.08-91.34)$ \\
Positive predictive value & 75.34 & $(64.77-85.91)$ \\
Negative predictive value & 92.31 & $(86.71-97.91)$ \\
Diagnostic accuracy & 80.75 & $(74.83-86.67)$ \\
\hline
\end{tabular}

\section{DISCUSSION}

The aim of our study has been to analyze at the initial diagnostic moment possible differences related to several parameters between cases with and without metachronous neoplastic colorectal lesions. Finding a higher number of these variables in cases where new neoplasms will be diagnosed could indicate a risk factor for the development of metachronous lesions. Cases with metachronous tumors (all of them with associated adenomas) were classified into the same group as metachronous adenomas, as others authors have published (8), due to the limited number of metachronous carcinomas in our series.

Our median endoscopic follow-up period of 48 months is in middle position in relation to other publications $(8,28,29)$, with a wide range, even reaching 112 months in one isolated case. Median diagnostic period until the first metachronous lesions was detected was 28 months. Nevertheless, in $9.3 \%$ patients the first metachronous adenoma was diagnosed more than 5 years after surgery. Consequently, the follow-up period was extended to the maximum possible, so as to detected the later metachronous lesions.

In the revised bibliography, we found several studies designed with a similar purpose (30-32). However, these authors analyzed a scarce number of variables. In a Spanish multicentric study published in 2007 (8), the reduced number of variables analyzed in previous studies is remarked, adding other possible risk factors, with a final number of 20 parameters analyzed. The aim of our study has been to identify a wider group of risk factors than previously published, including familial and personal history, patient clinical data, toxic habits, some particular drug consumption, initial anatomopathological neoplastic findings, comprising certain immunohistochemical markers expression. Thus, we have finally analyzed 40 possible risk factors, much more than considered in previous publications. Five of these 40 variables showed an independent prognostic value, statistically significant, as risk factors for development of metachronous adenomatous lesions. Alcohol intake, location of initial CRC distal to the splenic flexure, presence of synchronous adenomas (isolated and multiple) and presence of synchronous advanced adenoma seem to favor metachronicity. On the other hand, tumor MUC-5 expres- sion proved to have a protective role for developing metachronous lesions.

It has been published that alcohol intake increase the risk of developing colon neoplastic lesions $(29,32,33)$, even at a younger age (32). There are studies showing the relation between a continuing alcohol intake, including moderate doses of 20-40 g/day (34), with a higher frequency of colorectal neoplasms (adenomas and carcinomas), especially in males $(33,34)$. A higher proportion of synchronous adenomas have been described in patients with CRC and alcohol consumption (4). In addition, there is only one study where the lack of relation between alcohol intake and colonic adenomas recurrence is shown (35). A significant increase in metachronous adenomas incidence in patients with alcohol consumption is registered in our series. To our knowledge, this data has not been reported before and should be verified in later studies.

It has been discussed whether CRC location could have influenced on the incidence of metachronous lesions. Proximal CRC location and a tendency for multicentric lesions are described in patients with non polyposis hereditary colorectal cancer (3). Published results concerning carcinomas are contradictory and scarce, having referred both, the lack of correlation between initial tumor location and metachronicity (36), and the increased incidence of metachronous lesions in proximal $(37,38)$ and distal cancers $(32,39)$ as well. All of these studies are focused on metachronous carcinomas and the possible effect on the appearance of new adenomas, which is more frequent, is not investigated. Analyzing our series, we found a significant higher proportion of metachronous lesions in patients with distal CRC. In the revised bibliography, there are no conclusive data which could clarify the association between CRC location and metachronicity. One possible explanation could be that surgeons usually perform a right hemicolectomy in proximal CRC, which means a longer colonic resection than in distal CRC, where a segmentary resection is recommended. Thus, when the length of the remnant colon is shorter, the probability of developing metachronous lesions decreases. An upward trend to a proximal location in successive metachronous adenomas generation is described, reinforcing this hypothesis $(40,41)$. A right hemicolectomy would decrease the proportion of metachronous adenomas, which trend to be located in the proximal colon. Synchronous adenomas in CRC are the expression of tumor multicentriciy. Once all the initial metachronous lesions are resected, this multicentricity could be responsible for the development of new metachronous lesions. Consequently, it is reasonable that the presence of synchronous adenomas could be a risk factor for developing metachronous neoplasms. Except for a Chinese study of 2005 (39), all the rest of revised publications confirm a higher metachronicity risk in patients with synchronous adenomas $(8,12,36)$. Our cases with synchronous adenomas showed a significantly higher frequency of metachronous lesions, agreeing with these studies. Presence of an isolated synchronous adenoma represents an independent risk factor for metachronicity, whereas having more 
than one adenoma increases the probability of developing metachronous lesions during follow-up.

A higher trend towards metachronicity has been registered in patients with adenomatous resected polyps with no CRC, if these adenomas were larger $(11,42)$, with vellous component $(42)$, or with high grade dysplasia $(11,43)$. Advanced adenomas share these three characteristics of being larger, with villous component and with high grade dysplasia. In our study, the risk of metachronicity for advanced adenoma was analyzed globally, rather than to study the size, histological type or dysplasia grade separately just to facilitate the statistical analysis. Patients with synchronous advanced adenoma showed a significantly higher frequency of metachronous lesions compared to the rest of analyzed cases.

Immunohistochemical studies in CRC have been directed to the detection of the origin of metastatic colonic lesions and CRC prognosis with discordant results $(44,45)$. We did not find any publication focused on the possible value of immunohistochemistry for the assessment of tumor multicentricity risk, which is the principal topic of our research.

In the immunohistochemical study, frequency of MUC-5 tissular expression was the only parameter related to metachronicity with a significant value. Thus, patients with positive tissular expression of MUC-5 have an independent decreased risk for having metachronous neoplasms. This observation should be corroborated by subsequent studies, due to the lack of bibliographic antecedents.

The next step of our research was to draw up an individual risk index so as to classify patients into one of the three different risk groups: low, medium and high risk group for metachronous lesions development. This classification has shown a high discrimination power for estimating metachronicity risk. In addition, we tried to compare our risk group classification to a prognostic test, which results would be "positive" or "negative" for metachronicity. The incidence of metachronous lesions in medium risk patients was similar to overall incidence rate, which allows considering the medium risk group identical to the global population of our series. This finding could be interpreted as a "non conclusive result" in a test, because the pre-test and post-test values would be practically the same.

When the medium risk group is eliminated because its results are considered as inconclusive, $25 \%$ of our patients are excluded from our classification risk method. Despite being aware of this limitation, we registered a 16-fold lower probability for metachronous lesions development in low risk patients, compared to the high risk group.

Dividing cases in high and low metachronicity risk groups has shown an acceptable prognostic sensibility of $75 \%$. Specificity value has been superior: $84 \%$, with a very high negative predictive value of $92 \%$. As a whole, prognostic accuracy value reached $81 \%$. This predictive accuracy value beats clearly the pre-test probability of developing metachronous lesions, which was $42.2 \%$ for the overall population of our series. Besides, a high remarkable value for no detecting metachronous lesions in the low risk group was registered.
According to these data, drawing up an individual risk index allows to calculate correctly the risk of developing recurrent neoplastic lesions at the time of surgical treatment. Classifying patients into their predictable risk group will facilitate to establish a more appropriate postoperative follow-up. Patients classified into the low risk group would need a less strict follow up when looking for metachronous lesions, if our findings were confirmed.

In the revised bibliography we could not find any other research related to draw up an individual risk index for development of metachronous lesions in CRC which combine epidemiological, clinical, histological, and immunohistochemical variables. On one hand, this fact confers originality to our study, but, on the other hand, it makes us to be cautious when considering our results in the clinical practice, due to the lack of references. Therefore, it would be necessary to validate our data by prospective studies, preferably multicentric, and with a programmed postoperative follow-up.

\section{REFERENCES}

1. Tárraga-López PJ. Should we prevent colorectal cancer? Rev Esp Enferm Dig 2011;103:503-7.

2. Binefa-Rodríguez G. Colorectal cancer mortality trends in Spain. What shall we do? Rev Esp Enferm Dig 2011;103:285-8.

3. Piñol V, Andreu M, Castells A, Paya A, Bessa X, Jover R. Synchronous colorectal neoplasms in patients with colorectal cancer: predisposing individual and familial factors. Dis Colon Rectum 2004;47:1192-2000.

4. Borda A, Martínez-Peñuela JM, Muñoz-Navas M, Prieto C, Betés M, Borda F. Synchronous neoplastic lesions in colorectal cancer. An analysis of possible risk factors favouring presentation. Rev Esp Enferm Dig 2008;100:139-45.

5. Van Stolk RW, Beck GJ, Baron JA, Haile R, Summers R. Polyp Prevention Study Group. Adenoma characteristics at first colonoscopy as predictors of adenoma recurrence and characteristics at follow-up. Gastroenterology 1998;115:13-8.

6. Chung DC, Rustgi AK. DNA mismatch repair and cancer. Gastroenterology 1995;109:1685-99.

7. Karoui M, Tresallet C, Brouquet A, Radvanyi H, Penna C. Colorectal carcinogenesis.1. Hereditary predisposition and colorectal cancer. J Chir 2007; 144:13-8

8. Ballesté B, Bessa X, Piñol V, Castellví-Bel S, Castells A, Alenda C, et al. Detection of metachronous neoplasm in the colorectal cancer patients. Identification of risk factors. Dis Colon Rectum 2007;50:971-80.

9. Perea J, Lomas M, Hidalgo M. Molecular basis of colorrectal cancer: towards an individualized management? Rev Esp Enferm Dig 2011;103:29-35

10. Bonithon-Kopp C, Fenger PF, Cabeza E, O Morain C, Kronborg O, Faivre J. Colorectal adenoma characteristics as predictors of recurrence. Dis Colon Rectum 2004;47:323-33.

11. Saini SD, Kim HM, Schoenfeld P. Incidence of advanced adenomas at surveillance colonoscopy in patients with a personal history of colon adenomas: a meta-analysis and systematic review. Gastrointest Endosc 2006;64:614-26.

12. Martinez ME, Baron BA, Lieberman DA, Schatzkin A, Lanza E, Winawer SJ, et al. A pooled analysis of advanced colo-rectal neoplasia diagnoses after colonoscopic polypectomy. Gastroenterology 2009;136:832-41.

13. Moon CM, Cheon JH, Choi EH, Kim ES, Park JJ, Han SY, et al. Advanced synchronous adenoma but not simple adenoma predicts the future development of metachronous neoplasia in patients with resected colorectal cancer. J Clin Gastroenterol 2010;44:495-501.

14. Bouvier AM, Latournerie M, Jooste V, Lepage C, Cottet V, Faivre J. The lifelong risk of metachronous colorectal cancer justifies long-term colonoscopic follow-up. Eur J Cancer 2008;44:522-7. 
15. Rajaratnam SG, Dennett ER. Development of metachronous neoplams after colorrectal cancer resection: absence of synchronous neoplams predicts a lower risk. N Z Med J 2009;122:61-6.

16. Erenay FS, Alagoz A, Banerjee R, Cima RR. Estimating the unknown parameters of the natural history of metachronous colorectal cancer using discrete-event simulation. Med Decis Making 2011;31:611-24.

17. Fukutomi Y, Moriwaki H, Nagase S, Tajika M, Naito T, Miwa Y, et al. Metachronous colon tumors: risk factors and rationale for the surveillance colonoscopy after initial polypectomy. J Cancer Res Clin Oncol 2002;128:569-74.

18. Griffioen G, Bosman FT, Verspaget HW, Sier KF, Biemond I, Lamers CB. Comparative evaluation of carcinoembryonic antigen, secretory component, and mucins in index and metachronous adenomas of the colorectum. Gastroenterology 1991;101:919-26.

19. Rex DK, Kahi CJ, Levin B, Smith RA, Bond JH, Brooks D, et al. Guidelines for colonoscopy surveillance after cancer resection: a consensus update by the American Cancer Society and the US Multi-Society Task Force on Colorectal Cancer. Gastroenterology 2006;130:1865-71.

20. Tjandra JJ, Chan MK. Follow-up after curative resection of colorectal cancer: a meta-analysis. Dis Colon Rectum 2007;50:1783-99.

21. Prevención del cáncer colorrectal. Guía de Práctica Clínica. Grupo de trabajo de la guía de práctica clínica de prevención del cáncer colorrectal. Actualización 2009. Asociación Española de Gastroenterología, Sociedad Española de Medicina de Familia y Comunitaria, y Centro Cochrane Iberoamericano. Programa de Elaboración de Guías de Práctica Clínica en Enfermedades Digestivas, desde la Atención Primaria a la Especializada: 4. Barcelona: Elsevier España, S.L.; 2009.

22. Søreide K. Endoscopic surveillance after curative surgery for sporadic colorectal cancer: patient tailored, tumor targeted or biology-driven? Scand J Gastroenterol 2010;45:1255-61.

23. Demeter JG, Freeark RJ. The role of prophylactic subtotal colectomy in metachronous carcinoma of the colon and rectum. Surg Gynecol Obstet 1992;175:1-7.

24. Vasen HF, Mecklin JP, Khan PM, Lynch HT. The International Collaborative Group on Hereditary Non-Polyposis Colorectal Cancer (IGCHNPCC). Dis Colon Rectum 1991;34:424-5.

25. Vasen HF, Watson P, Mecklin JP, Lynch HT. New Clinical criteria for hereditary nonpolyposis colorectal cancer (HNPCC, Lynch Syndrome) proposed by the International Collaborative Group on HNPCC. Gastroenterology 1999;116:1453-6.

26. Umar A, Boland CR, Terdiman JP, Syngal S, de la Chapelle A, Rüschoff J, et al. Revised Bethesda Guidelines for hereditary non-polyposis colorectal cancer (Lynch syndrome) and microsatellite instability. J Natl Cancer Inst 2004;96:261-8.

27. Greene FL, Page DL, Fleming ID, editors. AJCC (American Joint Committee on Cancer) Cancer Staging Manual. 6th ed. New York: SpringerVerlag; 2002. p. 113.

28. Shitoh K, Konishi F, Miyakura Y, Togashi K, Okamoto T, Nagai H. Microsatellite instability as a marker in predicting metachronous multiple colorectal carcinomas after surgery: a cohort-like study. Dis Colon Rectum 2002;45:329-33.

29. Cho JH, Lee SK, Kim TI, Kim WH. Characteristics and risk factors of synchronous and metachronous polyp in colorectal cancer. Korean J Gastroenterol 2004;43:168-75.
30. Masubuchi S, Konishi F, Togashi K, Okamoto T, Senba S, Shitoh K, et al. The significance of microsatellite instability in predicting the development of metachronous multiple colorectal carcinomas in patients with non-familial colorectal carcinoma. Cancer 1999;85:1917-24.

31. Tsantilas D, Ntinas A, Petras P, Zambas N, Al Mogrambi S, Frangandreas S, et al. Metachronous colorectal adenocarcinomas. Tech Coloproctol 2004;8:202-4.

32. Rennert G, Robinson E, Rennert HS, Neugut AI. Clinical characteristics of metachronous colorectal tumors. Int J Cancer 1995;60:743-7.

33. Mizoue T, Tanaka K, Tsuji I, Wakai K, Nagata C, Otani T, et al. Alcohol drinking and colorectal cancer risk: an evaluation based on a systematic review of epidemiological evidence among the Japanese population. Jpn J Clin Oncol 2006;36:582-97.

34. Otani T, Iwasaki M, Yamamoto S, Sobue T, Hanaoka T, Inoue M, et al. Alcohol consumption, smoking, and subsequent risk of colorectal cancer in middle-aged and elderly Japanese men and women: Japan Public Health Center-based prospective study. Cancer Epidemiol Biomarkers Prev 2003;12:1492-500.

35. Jacobson JS, Neugut AI, Murray T, Garbowski GC, Forde KA, Treat MR, et al. Cigarette smoking and other behavioral risk factors to recurrence of colorectal adenomatous polyps (New York City, NY, USA). Cancer Causes Control 1994;5:215-20

36. Yamazaki T, Takii Y, Okamoto H, Sakai Y, Hatakeyama K. What is the risk factor for metachronous colorectal carcinoma? Dis Colon Rectum 1997;40:935-8.

37. Gervaz P, Bucher P, Neyroud-Caspar I, Soravia C, Morel P. Proximal location of colon cancer is a risk factor for the development of metachronous colorectal cancer: a population-based study. Dis Colon Rectum 2005;48:227-32.

38. Legget BA, Cornwell M, Thomas LR, Buttenshaw RL, Searle J, Young $\mathrm{J}$, et al. Characteristics of metachronous colorectal carcinoma occurring despite colonoscopic surveillance. Dis Colon Rectum 1997;40:603-8.

39. Lan YT, Lin JK, Li AF, Lin TC, Chen WS, Jiang JK, et al. Metachronous colorectal cancer: Necessity of post-operative colonoscopic surveillance. Int J Colorectal Dis 2005;20:121-5.

40. Nusko G, Hahn EG, Mansmann U. Characteristics of metachronous colorectal adenomas found during long-term follow-up: analysis of four subsequent generations of adenoma recurrence. Scand J Gastroenterol 2009;44:736-44.

41. Yamaji Y, Mitsushima T, Yoshida H, Watabe H, Okamoto M, Ikuma H. et al Right-sided shift of metachronous colorectal adenomas after polypectomy. Scand J Gastroenterol 2007;42:1466-72.

42. Noshirwani KC, van Stolk RU, Rybicki LA, Beck GJ. Adenoma size and number are predictive of adenoma recurrence: implications for surveillance colonoscopy. Gastrointest Endosc 2000;51:433-7.

43. Demetriades H, Kanellos I, Blouhos K, Tsachalis T, Vasiliadis K, Pramateftakis MG, et al. Synchronous polyps in patients with colorectal cancer. Tech Coloproctol 2004;8:72-5.

44. Chivukula M, Bhargava R, Dabbs DJ. Diagnostic, theragnostic and genomic immunohistochemistry: Standardization issues. Adv Anat Pathol 2008;15:302-4.

45. Lee WS, Park S, Lee WY, Yun SH, Chun HK. Clinical impact of tumorinfiltrating lymphocytes for survival in stage II colon cancer. Cancer 2010;116:188-99. 